\title{
Bioanalysis
}

\section{Future trends in outsourcing: a summary of the Bioanalysis Zone Survey}

\author{
"It will be interesting to see how the changes we are currently \\ seeing shape the bioanalytical arena of the future..."
}

\section{Future trends in outsourcing}

As the bioanalytical field continues to grow exponentially with the development of more complex molecules and as more Pharma continue to reduce their internal capacities for $\mathrm{R} \& \mathrm{D}$, there has been an increasing demand to outsource work. To explore this further, Bioanalysis Zone featured a Spotlight on the Future Trends in Outsourcing earlier this year [1]. As part of this spotlight, the current models of outsourcing employed by Pharma were examined and experts within the field were consulted on how outsourcing is likely to change in the next 10 years and what these changes will mean for the CRO-Pharma relationship.

In order to gain a better understanding of how outsourcing is changing, a targeted survey was carried out to identify how the key players in industry are dealing with the changes in the industry. The full results of the survey are presented in an infographic online [2]. The survey was made up of 100 experts from around the world, consisting of 51 experts with a CRO background, 44 experts working in Pharma and Biotech, and the remaining 5 experts in academia. The results of the survey were then analyzed by three independent experts: Bruce Stouffer (Executive Director Immunochemistry, PPDI), Erik Burns (Division Chair, Assistant Dean of Outreach \& Professional Development, University of WisconsinMadison, USA) and Randall Guthrie (Vice President, Pyxant Labs, Inc.) during a panel discussion [3]. Here, the experts discussed whether the results of the survey reflected their own experience in the industry, gave their insight into why these changes are happening and how best to optimize the interface between CROs and Pharma to ensure best practice and scientific progress.
Changes in bioanalytical outsourcing

The first section of the survey addressed the changes in outsourcing between 2014 and 2016 from a CRO perspective. It was observed that $58 \%$ of the respondents from CROs found that the number of samples they were receiving had increased compared with $9 \%$ who found the number of samples had decreased over the last 2 years, while the remainder found no change in sample size (Figure 1). The respondents who observed the increase in sample size cited that investments in automation, instruments and additional staff had improved efficiency for managing the increase in samples being processed.

This upward trend is likely to increase in the next few years and when asked to predict the changes in outsourcing in the next 2 years, the consensus of the survey was that the percentage of outsourcing to CROs would generally increase: a thought also reflected by our expert panelists. This was believed to be due to an increase in the talent and the level of expertise within CROs, which has increased over the last few decades. We are also beginning to see CROs becoming experts in certain fields such as biologics and immuneoncology. From the Pharma perspective, an increase in outsourcing could be attributed to the current shift toward Pharma devoting more of their staff to $\mathrm{R} \& \mathrm{D}$, in addition for economic reasons. Touching upon whether CROs are equipped for this increase, our panelists explained that this varied dramatically between CROs, owing to the differences in resource and technology capability of different CROs and the difficulty in predicting the variable workload.

\section{The CRO-Pharma relationship}

The second section of the survey was aimed toward the CRO-Pharma relationship,
Amy Craddock*,1 \& Sankeetha Nadarajah ${ }^{1}$

'Future Science Group, Unitec House, 2 Albert Place, London N3 1QB, UK

*Author for correspondence:

a.craddock@future-science.com 


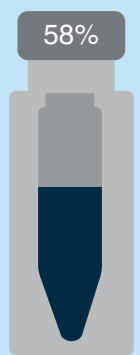

Increased

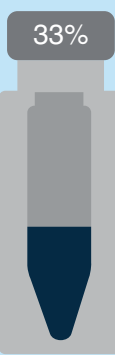

Remained similar

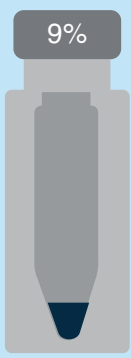

Decreased
Figure 1. Has the number of samples received by CROs for analysis changed in the last 2 years?

establishing whether the expectations from both perspectives are aligned (Figure 2). Here, we asked experts with Pharma backgrounds to rank the most important criteria for choosing a CRO to work with, while asking the experts from CROs what they believed were the most important criteria for Pharma. This revealed some interesting discrepancies between the two. Most notably, CROs underestimated the importance of cost for Pharma, with $75 \%$ of our Pharma respondents ranking cost as the most important factor in choosing a CRO, in comparison to only $50 \%$ of experts from CROs.

This was remarked upon in the panel discussion, where all three experts expressed their surprise that cost was prioritized above speed, quality and flexibility. This led to a discussion on two points, first, whether the CRO model needs revising and second whether the emphasis on the current CRO model lies too heavily on the bottom line rather than focusing on scientific integrity and the advancement of science. It was identified that without a clearly defined and aligned vision between CRO and Pharma, it is inevitable that difficulties will arise. It was also considered that there should really be a greater onus on quality, which should be the most important factor for both CRO and Pharma, since if you compromise on quality it will ultimately cost more in the long run. From a business perspective, the panelists explored how the expectations need to be aligned ahead of time in order to ensure best practice. Potential barriers that are preventing a strong CROPharma relationship were identified to include money, the intellectual property for Pharma and the quality that the CROs are able to deliver.

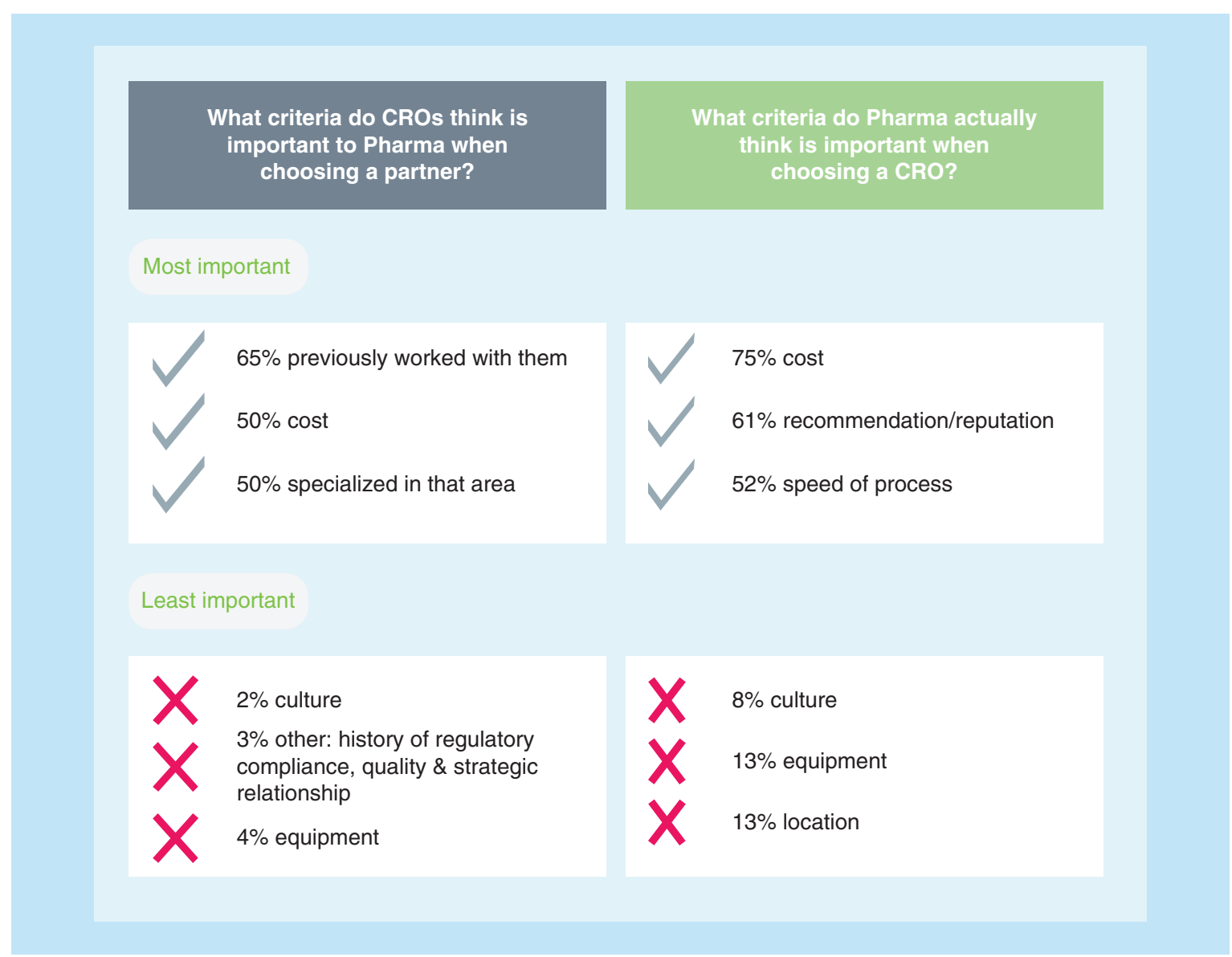

Figure 2. Criteria for CRO selection by Pharma: CRO and Pharma perspective. 
Another key finding from the survey was on whether there is a rightful demand for Pharma to share all aspects of clinical information with the CRO (Figure 3). Eighty percent of respondents thought that it was important for Pharma to share expertise and knowledge (of these respondents, $64 \%$ said yes to some extent while remaining $16 \%$ said yes completely). This highlights the importance of communication between the CRO and Pharma, which leads us on to the final section of the survey on how to optimize the CRO-Pharma interface. Overwhelmingly, the key responses for how best to optimize the relationship between CRO and Pharma involved strengthening communication and ensuring an understanding of what the expectations are between sponsor and CRO.

One of the key points identified by experts in the survey panel discussion was that the engagement within the $\mathrm{CRO}$ workforce is absolutely critical. It is important to keep the workforce engaged in the work they are doing and making sure that they are contributing to important areas of drug discovery and development. Therefore, it is essential that the CROs are aware of the work they are doing. This point reflects a similar view on the Changing World of Bioanalysis panel discussion [4] and European Bioanalysis Forum (EBF) address panel discussion (also featured on the Zone) [5]. During both discussions, the need for more scientific interactions between CRO and Pharma was strongly emphasized. The EBF panelists addressed the changing relationship that has been observed between CRO and Pharma as a direct result of the increase in outsourcing and an increasingly global team. The shift from CROs being considered as a service provider to being an extension of the internal laboratory in partnership with the Pharma was discussed, as well as the need for transparency between $\mathrm{CRO}$ and Pharma to allow for more efficient progress (including, sharing of data, operating procedures and strategy). The importance of having confer-

\section{References}

1 Bioanalysis Zone. Spotlight on future trends in outsourcing. www.bioanalysis-zone.com/category/spotlights/

2 Bioanalysis Zone. Future trends in outsourcing survey infographic.

www.bioanalysis-zone.com/2017/03/13/spotlfuture

3 Bioanalysis Zone. Are Pharma and CROs really on the same page with regard to outsourcing?

www.bioanalysis-zone.com/2017/03/24/pharma-cros

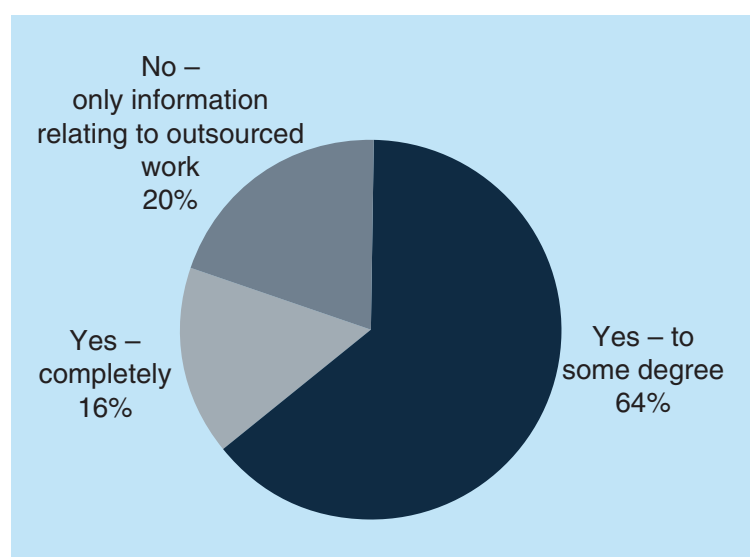

Figure 3. Is there a rightful demand for Pharma to share all aspects of clinical information of a particular project to CROs?

ences to bring together the entire community from all over the world to encourage communication and harmonization was also highlighted.

\section{Conclusion}

As the bioanalytical field continues to evolve with the development of more complex molecules and the demand to outsource work continues to increase, models and trends in outsourcing are also likely to evolve in the next few years. It will be interesting to see how the changes we are currently seeing shape the bioanalytical arena of the future, including the quality of work, models, trends and working relationships.

\section{Financial \& competing interests disclosure}

A Craddock \& S Nadarajah are employees of Future Science Ltd. The authors have no other relevant affiliations or financial involvement with any organization or entity with a financial interest in or financial conflict with the subject matter or materials discussed in the manuscript apart from those disclosed.

No writing assistance was utilized in the production of this manuscript.
Bioanalysis Zone. The changing world of bioanalysis: how do we embed science in the Pharma-CRO partnership? www.bioanalysis-zone.com/2017/02/24/embed-

5 Bioanalysis Zone. EBF addresses: the evolving relationship between $\mathrm{CRO}$ and Pharma.

www.bioanalysis-zone.com/2017/03/23/ebf2016panel-ebf/ 